\title{
The artificial intelligence and design of multibody systems with predicted dynamic behavior
}

\author{
Vladimir Poliakov \\ Russian University of Transport (RUT - MIIT), \\ 9b9 Obrazcova Street, Moscow, 127994, \\ Russia
}

Received: October 26, 2020. Revised: November 27, 2020. Accepted: December 12, 2020. Published: December 14, 2020.

\begin{abstract}
The problem of complicated dynamic system optimization is very difficult for human intellect. The design of these systems comprises typical tasks of artificial intelligence big data analysis, decision making, etc. In this article, we applied artificial intelligence approach to optimize the properties of the multibody dynamic system. It is very important to study the whole carrying system of a high-speed railroad in its integrity because the elements of the system interact and influence each other simultaneously. The system should include the train of several cars, the track upper structure, and the bridges. It is possible to synthesize the optimal system with predicted behavior that meets various constraints on dynamic parameters and interaction between the elements of the system.
\end{abstract}

Keywords-artificial intelligence, decision support systems, dynamical system, modeling and simulation, optimal control.

\section{INTRODUCTION}

The trains and the track are integral parts of railroads. The bridges length may reach up to $95 \%$ of the total length of a high-speed railroad, so we need to explore the triple system of the "Bridge-Track-Car System" (BTCS). The trend analysis during optimization is necessary to determine the direction and resources of optimizing a system. Therefore, scientific methods of decision-making are necessary that will lead us to decision support system. It requires a great amount of information analysis dealing with behavior and changes of BTCS that consists of mechanisms and structures.

Usually the three above mentioned parts of the BTCS are studied in different disciplines. It makes the research of interactions between the parts difficult. This interaction is important for running safety (wheel-rail contact) and track endurance (rail bed loading) for example. Therefore, attempts to get over the so called hipper-specialisation were undertaken in [1]-[3]. The researchers take into account only one (but important) aspect: modelling of coupled dynamic behaviour of BTCS. However, the objective is optimal system synthesis, where dynamic interaction is just one aspect of the design problem. In computer modeling the behavior of a definite system with certain parameters is envisaged, but the practical problem is to appoint these parameters. It is very important if we want to reach certain goals for a system.

\section{ARTIFICIAL INTELLIGENCE APPROACH}

Artificial intelligence (AI) approach could be very useful in this case. Today AI is still young science and it deals with important theoretical problems. There are aspects of reasoning, scientific decision making of system optimization [4], knowledge processing and self-learning [5], [6], fuzzy logic [7], [8] and genetic algorithms [9], [10]. The interesting approach combining optimization with genetic algorithm was proposed in [11]. It is interesting to underline, that AI methodology is used in different faculties. It makes AI a kind of modern philosophy. Practical application of AI methods in engineering are at the initial stage. AI is used for traffic control, logistics processes, advertising, and so on. However, the design of complicated systems, especially a multibody dynamic system, urgently requires decision-support methods [12].

The term 'artificial intelligence' was introduced by John McCarthy in 1959 [13]. In this article, AI was considered just as a computational system, that could be able to create subroutines [13]. Later, John McCarthy clarified that 
'Intelligence is the computational part of the ability to achieve goals in the world' [14]. The BTCS is a teleological system. The goals of the BTCS are the following:

- safe and comfort passing of a train through a bridge zone;

- maximization of durability of the most wearing part (track);

- decrease of mass and materials-output ratio for the most expensive element (bridge beams).

The theory of optimal control could be very useful in this case. The review in this area could be found in [15]. It is also important to develop the design methodology that uses the solution for complicated structures in closed form (see for example [16], [17]). In our research, the goals of the BTCS system denote extremal value of optimality criteria. These goals are achieved by changing the dynamic properties of the beams and track along their length using control functions. The theory of optimal control implies the approach that minimizes a pre-determined criterion with certain constraints fulfilled [18]. In this theory, control functions are changed in time domain. Those functions do not suit buildings and structures, because they should remain stable for centuries as opposed to mechanisms that are designed for motion. For example, the Milvio bridge in Rome was built in 109 BC and it is still in operation. Therefore, we suggest the creation of control functions that change in space. The goals are coupled with those constraints fulfilment. The vector-specified criteria of optimization and vibration equations take these control functions into account. For instance, the minimal mass of the bridge beams criterion [18] looks like (1):

$$
\sum_{k=1}^{n} \int_{0}^{l_{k}} \alpha^{2}\left(x_{k}\right) \rho F_{0} d x_{k} \rightarrow \min
$$

where $\alpha(x)$ is the sought control function, $\rho$ - material density, $F_{0}$ - the starting value of the cross section area on the first iteration, $n$ - the number of the bridge spans of $l_{k}$ length each. The equation of bridge beam vibration is (2):

$E_{b} J_{o} \frac{\partial^{2}}{\partial x_{h}^{2}}\left(\alpha^{4}\left(x_{b}\right) \frac{\partial^{2} y_{b}}{\partial x_{h}^{2}}\right)+\alpha^{2}\left(x_{b}\right) \rho F_{0} \frac{\partial^{2} y_{b}}{\partial t^{2}}+\mathrm{c} \frac{\partial y_{b}}{\partial t}=$

$\mathrm{c}_{\mathrm{p}}\left(\frac{\partial y_{\mathrm{p}}}{\partial t}-\frac{\partial y_{b}}{\partial t}\right)+\gamma(x) \beta(x) U^{\prime}\left(y_{\mathrm{p}}-y_{b}\right)$

with the zero boundary and initial conditions, $y_{p}, y_{b}-$ rail and beam displacement accordingly, $J_{0}$ - starting value of the cross section inertia, $c$ - the beam damping factor.

The equation (2) is coupled with partial equation of rail vibration [19]:

$\left(k_{b} \delta(x) \rho_{p} F_{p}\right) \frac{\partial^{2} y_{p}}{\partial t^{2}}+c_{\mathrm{p}}\left(\frac{\partial y_{\mathrm{p}}}{\partial t}-\frac{\partial y_{b}}{\partial t}\right)+$

$E_{p} J_{p} \frac{\partial^{4} y_{p}}{\partial x^{4}}+\gamma(x) \beta(x) U^{\prime}\left(y_{\mathrm{p}}-y_{b}\right)=P(x, t)$

with the zero boundary and initial conditions, where $P(x, t)$ is wheel-rail vertical contact force, $\gamma(x), \beta(x)$ are track control functions. The train vibration is described by ordinary differential equations that determine $P(x, t)$ together with equation (3). It is a significant difference against other approaches [20],[21], where $P(x, t)$ is a predicted function, but not the result of interaction within the system or even $P$ is constant [22], [23]. In some AI applications, the interaction forces $P$ are not envisaged in optimizing [24].

It is possible to determine the control functions by means of minimizing the criterion (1). Thus, they should not be inputted. The developed approach based on the integral carrier BTCS permits to create the multibody system with predicted dynamic behaviour. The incorporation of the elements into integrated BTCS is ensured by equations of coupled vibration $(2,3)$. It makes some foundation for AI implementation in railroad design development.

Other criteria were implemented for track optimization [18]. For instance, the criterion of minimal deviation of rail bed load in reference to predicted value looks like (4):

$D=\int_{L} \int_{T}\left(\gamma(x) \beta(x) U^{\prime}\left(y_{\mathrm{p}}-y_{b}\right)-Q\right)^{2} d x d t \rightarrow \min$

where $\gamma(x)$ and $\beta(x)$ are control functions, $U^{\prime}$ is rail bed stiffness, $Q$ is predicted value of rail bed load, $L$ is the length of the track section including the bridge and transition zones, $T$ is the time of train passing. This criterion leads to the decrease of local rail bed overload and increases endurance of the track. Moreover, this criterion allows decreasing average rail bed load to increase endurance.

Solution of the vibration equations was made by the socalled finite-difference method and the Euler explicit scheme which assumes a very small time step $(0.0001 \mathrm{~s})$ to achieve stability of the computational process. Thus, this scheme allows concerning vibration frequency up to $5 \mathrm{kHz}$.

The optimization is coupled with inequation constraint fulfilment that is associated with technology requirements, running safety, comfort, displacement, etc. The running safety constraint prevents derailment during $T$ time on $L$ space (see the explanations to expression (4)) and looks like:

$$
P_{\text {min }}>P_{\text {lim }}
$$

where $P_{\text {lim }}$ is sufficient value of the vertical force in wheel-rail contact.

Predicted behavior of the multibody BTCS is achieved by:

- precise description of the vibration of a car body, bogies, wheelsets, track, and beams using a system of equations (2), (3);

- including controlling functions into both the vibration equations (2), (3) and the criteria (1), (4);

- determination of the controlling functions $\alpha(x), \beta(x), \gamma(x)$, that make different criteria (1), (4) extreme;

- achievement of the predicted extreme volume of vibration parameters (displacement, bending moment, acceleration, etc.);

- certain parameters that do not exceed the predicted value (wheel-rail contact forces $P$, internal forces if they are not in the concerned criterion, etc.).

The optimization process is shown schematically in Fig.1. 


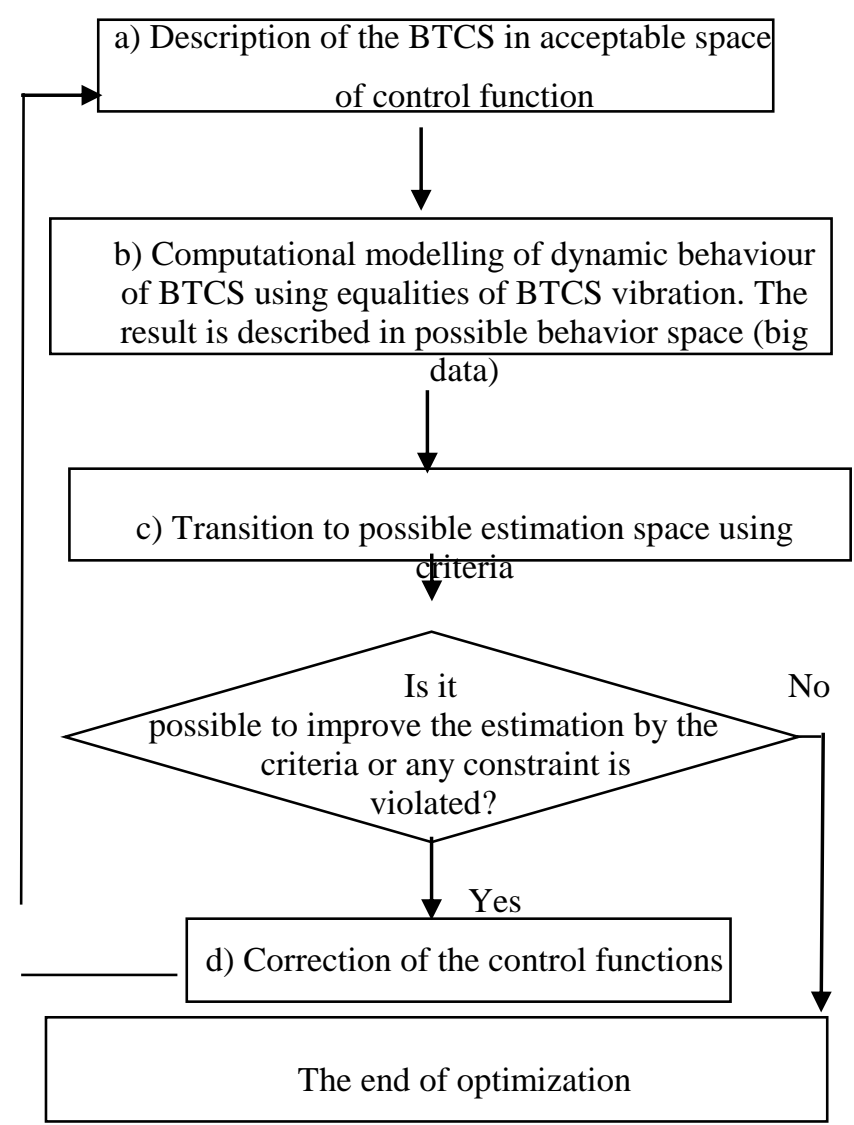

Fig. 1 The scheme of solving the system optimization

The AI features appear at two stages of the algorithm (Fig.1). It was mentioned above, that usually research takes into account only one aspect: modelling of coupled dynamic behaviour of BTCS (step ' $b$ ' in Fig.1). However, it is just a part of the problem shown in Fig.1. The first stage is the analysis of trends in dynamic behavior of the BTCS and sensitivity of the BTCS to variations of control. The analysis deals with two problems. First, the description of the BTCS vibration is performed in multidimensional (more than 200 dimensions) behavior space. Second, the description is carried out every $0.0001 \mathrm{~s}$ while the train is passing through the bridge zone because of the requirement of the computational process robustness. Moreover, wheel-rail contact force that is characterized by high-frequency vibration, has to be checked according to running safety constraint. Both problems create a huge amount of data (big data). The second stage where the AI features appear is the definition of the ability to continue the optimization in multi-criteria space and direction of control functions changes. The human intellect cannot operate the big data in multidimensional spaces.

AI methodology, i.e. particle swarm optimization (PSO) [25]) was used for demonstration of global extremum reaching.

\section{DISCUSSION OF THE OPTIMAL DESIGN RESULTS}

During optimization, it is possible to reach the optimal dynamic parameters that provide wheel-rail contact safety to prevent derailment, material efficiency of the bridge superstructure, comfort of passengers and ballast wearing. All the following results were achieved by the original author's program. The optimization allows:

- Decreasing the mass of a bridge superstructure up to $20 \%$ compared to conventional design. For instance, the total mass of the $50 \mathrm{~m}$ two-ways beam bridge superstructure before optimization was $1.8 \mathrm{kt}$ and after optimization it decreased to 1.5kt [18] (Fig. 2) simultaneously with constraints fulfillment. In Fig. 2, the outermost cross sections are not shown. We would like to point out that the outermost cross sections can take any dimensions (for instance, for constructive reasons) because they have little effect on the dynamic behavior of the beam. The beam rigidity decreases correspondingly to beam mass, but both parameters do not change identically along the superstructure. The changes of the $\alpha(x)$ control function are more significant in the middle of the beams, in order to comply with the constraint that keeps the maximum bending moment value constant (Fig. 3). At the beginning of the optimization process, the bending moment value is increasing up to the seventh iteration by $4 \%$ to the starting value, later it is decreasing by $1 \%$ to the starting value and finally stabilizes near the starting value $\left(0.99 \mathrm{M}_{1}\right)$.

- Decreasing the ballast local overload to the value that prevents ballast draft and decrease of the profile irregularity in the bridge zone including transitions. Fig.4 shows changing of the sleepers loading $Q_{2-2}$ just under the second wheel of the second car for the model problem (a train of three cars) before optimization ( $1^{\text {st }}$ iteration) and after optimization $\left(6^{\text {th }}\right.$ iteration). The standard deviation of railway sleeper loading for the real train (ten cars) is reduced by two times (from $2.8 \mathrm{kN}$ to $1.4 \mathrm{kN}$ ) providing evener loading to prevent the track derangement. Moreover, optimization decreases the maximum value of the loading lower than the critical value of $31 \mathrm{kN}$ (from $40 \mathrm{kN}$ to $30.7 \mathrm{kN}$ ) [19].

- Guaranteeing wheel motion across the bridge without uplift and decrease of derailment probability. Fig.5 shows the vertical force in the second wheel-rail contact of the second car for the model problem (three cars). One can see the twin wheel uplift (at $0.57 \mathrm{~s}$ and $0.63 \mathrm{~s}$ ) that means possibility of derailment. Fig.6 shows the situation for the real train as a whole. The minimal value during the train passing the bridge zone (including transitions) before the optimization was equal to 0 , which means uplift (Fig.6). In this case, $P_{\min }$ does not meet the constraint (5). The AI technology allows providing minimization of the criterion (4) and fulfillment of constraint (5) simultaneously. After the optimization, it becomes $24.6 \mathrm{kN}$ (the minimal permitted value $P_{\lim }$ is $23.8 \mathrm{kN}$ [19]).

- Providing good comfort while the train is moving on the bridge zone (Fig. 7) [18]. According to Eurocode [26], good comfort for passengers is obtained while the car superstructure acceleration is lower than $1 \mathrm{~m} / \mathrm{s}^{2}$. 




Fig. 2 The mass distribution of superstructure before optimization (red line) and after (green line)



Fig. 3 The maximum value of bending moment evolution during optimization.

Time, $s$

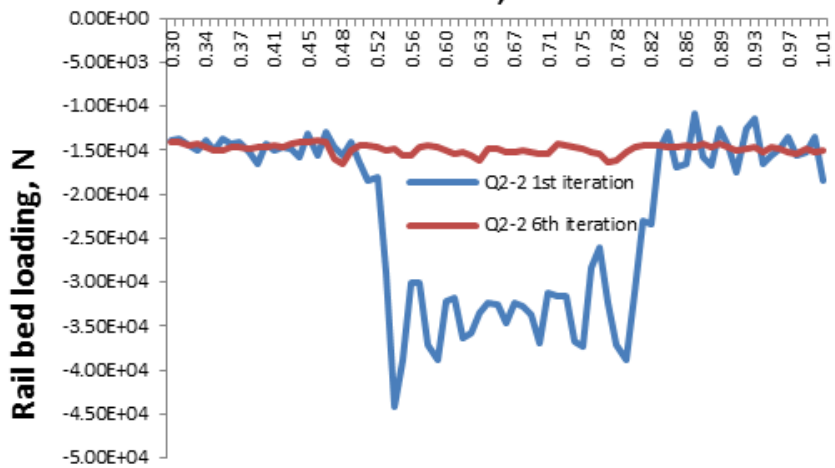

Fig.4 Loading on sleepers in the bridge zone before and after the optimization

Demonstration of global extremum reached during the optimization is a very important question. In this research, the modified particle swarm optimization (PSO) was used. Usually this method is used for parallel computing. In this research subsequent computing with a single central processor was used. This method can demonstrate global minimum of criterion reached when the optimization starts from different points in acceptable space of control function (Fig.1). Despite the different starting points of the optimization according to minimum bending moment criterion the optimization process leads to the decision in certain environs of the possible estimation space (Fig.1). It is shown in Table I. Obviously, the optimization lets avoidance of resonance (two bottom lines)

The behavior of a structure with some initial parameters of may not meet the constraints. It may turn out only during the optimization. In this case, the AI allows creating the optimal system that may have the criterion value that is worse than before the optimization, but the behavior of a structure meets the constraints, because the inequation constraints must strictly be carried out. The constraints have priority in comparison with optimality criteria because they connect with safety and technology possibilities. The example below explains this claim. Table II shows the considerable violation of the deflection constraint $(0.025 \mathrm{~m})$ at the beginning of the optimization (at the first iteration). This constraint holds at $18^{\text {th }}$ iteration by the increase of the control function, mass and stiffness of the beam in spite of the optimization criterion (1) which demands reducing the mass of the beam. The control function $\alpha(x)$ significantly increases up to 1.76 times. It leads to increase of the mass and stiffness of the beam instead of reducing them. It allows fulfilment the deflection of the constraint $0.025 \mathrm{~m}$.

TABLE I. OPTIMIZATION FROM DIFFERENT INITIAL POINTS LEADS TO THE SINGLE DECISSION

\begin{tabular}{|c|c|c|c|}
\hline $\begin{array}{c}\text { Beam } \\
\text { stiffness } \mathbf{E} \mathbf{J}_{\mathbf{0}} \\
\text { before } \\
\text { optimization, } \\
\mathbf{1 0}^{\mathbf{9}} \mathbf{~ N m}^{\mathbf{2}}\end{array}$ & $\begin{array}{c}\text { Beam mass } \\
\text { before } \\
\text { optimization, } \\
\mathbf{t} / \mathbf{m}\end{array}$ & $\begin{array}{c}\text { Bending } \\
\text { moment } \\
\text { index before } \\
\text { optimization, } \\
\mathbf{1 0}^{\mathbf{6}} \mathbf{~ N m}\end{array}$ & $\begin{array}{c}\text { Bending } \\
\text { moment } \\
\text { index after } \\
\text { optimization, } \\
\mathbf{1 0}^{\mathbf{6}} \mathbf{~ N m}\end{array}$ \\
\hline 4 & 2.40 & 2.25 & 1.68 \\
\hline 5 & 2.70 & 2.68 & 1.69 \\
\hline 6 & 2.90 & 3.31 & 1.68 \\
\hline $\mathbf{1 0}$ & $\mathbf{3 . 8 0}$ & $\mathbf{9 . 7 1}$ & $\mathbf{1 . 6 9}$ \\
\hline $\mathbf{1 1}$ & $\mathbf{3 . 9 0}$ & $\mathbf{9 . 8 2}$ & $\mathbf{1 . 6 7}$ \\
\hline
\end{tabular}

The objective of achieving minimal mass and rigidity of the bridge beam was formulated together with the constraint of maximum middle point deflection lower than $0.025 \mathrm{~m}$. In the first iteration, deflection reaches almost $0.2 \mathrm{~m}$ (Table II) and $\alpha(x)$ controlling function is constant and equal to 1 , but to the seventh iteration the deflection is closer to the constraint and later it will be lower than the constraint. It is achieved by significant controlling function increase. However, mass criterion in the $40^{\text {th }}$ iteration appears to be significantly worse, but the deflection constraint was carried out. This solution is conditionally optimal according to (1) and it is confirmed by decreasing of the $\alpha(x)$ controlling function in the near abutment cross sections which properties have insignificant impact on the middle point deflection.

TABLE II. CONSTRAINT FULFILLMENT DURING OPTIMIZATION

\begin{tabular}{|c|c|c|}
\hline Iteration & $\begin{array}{c}\text { Maximum beam } \\
\text { displacement, } \boldsymbol{m}\end{array}$ & $\begin{array}{c}\text { Maximum control } \\
\text { function value }\end{array}$ \\
\hline 1 & 0.1976 & 1.0 \\
\hline 2 & 0.0909 & 1.32 \\
\hline 3 & 0.0733 & 1.45 \\
\hline 4 & 0.0622 & 1.45 \\
\hline 5 & 0.0448 & 1.60 \\
\hline 6 & 0.0333 & 1.72 \\
\hline 7 & 0.0273 & 1.72 \\
\hline$\ldots$ & $\ldots$ & $\ldots$ \\
\hline 18 & 0.0243 & 1.76 \\
\hline
\end{tabular}


Another example concerning the constraints deals with running safety. The vertical force in wheel-rail contact must be more than minimum, which guarantees absence of wheel liftoff and prevents derailment. Therefore, we will optimize the BTCS according to criterion (4) with constraint (5) $\left.P(x, t)\right|_{\mathrm{T}}>$ $P_{\min }=23.8 \mathrm{kN}$, where $\mathrm{T}$ is the time of train passing through the bridge zone including transitions. This is an unsolvable problem without AI technology - big data analysis and decision-support.

Fig.6 shows change of optimality criterion $D(4)$, standard deviation of rail bed load and $P(x, t)_{\min }$ during optimization. The values of the parameters are scaled by casting-off the exponential parts of notation for inserting them to the common chart. It is seen that at the beginning of the optimization the constraint $\left.P(x, t)\right|_{\mathrm{T}}>P_{\min }=23.8 \mathrm{kN}$ is not kept because the liftoff of a wheel (or wheels) occurs (the vertical wheel-rail contact force decrease up to zero). This situation is very dangerous [28]. During the optimization, minimal value of $P(x, t)$ steadily goes up and to the $9^{\text {th }}$ iteration it becomes higher than the constraint of $23.8 \mathrm{kN}$ anywhere and anytime within $T$.

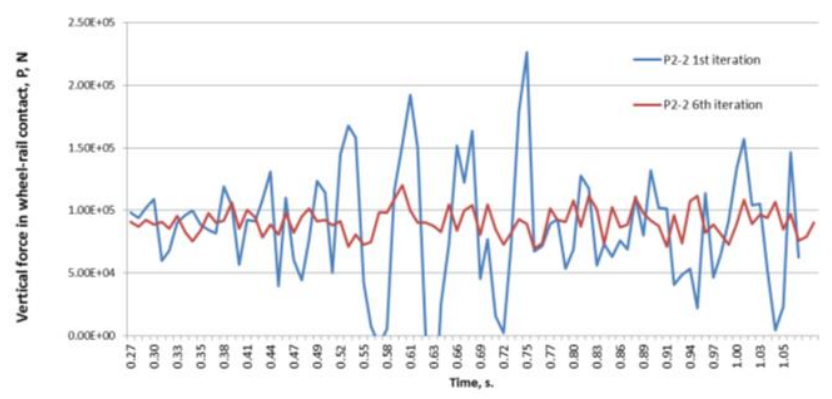

Fig.5 Wheel-rail contact force before and after the optimization



Fig. 6 The constraint makes optimization as conditionally optimal [27]



Fig. 7. Comfort control during the optimization
Absoluteness of the constraint (5) limits the functional $D$ (4) minimization. It is clearly seen after the second iteration. The other parameters behave in the same way.

\section{CONCLUSIONS}

The research showed the importance of the integral system dynamic analysis without application of numerous hypotheses of the interaction between different parts of the BTCS. Thus, the applied theory of optimal control of the carrier railway system with elements of artificial intelligence was developed. The applied theory allows designing the system with predicted behavior. In comparison with alternative methods, the theory allows securing running safety due to interaction forces evaluation by multibody system dynamics. It prevents derailment. The optimization of technical parameters instead of cost optimization makes the methodology independent from demand and supply on the market. Thus, unlike the traditional methodology of the preliminary design, the proposed approach allows reduction of the beam mass up to $20 \%$ that improves materials-output ratio. Concordant dynamics of the parts of the multibody system is possible due to AI methodology with the big data analysis.

The choice of the motion direction within many-dimensional behavior and estimation spaces by means of vector-specified controlling functions may be considered as a step forward to the development of artificial intelligence in civil engineering. The computer program can make decisions concerning control of technical parameters of the BTCS on the base of analysis of a great amount of information and a designer makes the final decision about the acceptability of the computer-aided design.

The developed theory allows maximization or minimization of certain parameters (beam mass, standard deviation of the rail bed loading, bending moment, etc.), forces other parameters to be equal to some predicted value (bending moment, maximal value of rail bed loading, etc.) and takes into account different constrains (minimal wheel-rail contact force, maximum beam deflection, car body acceleration, etc.).

In future, the presented approach may be extended to wider types of structures. Now it is suitable only for simply supported beams, that are nevertheless most widespread.

\section{ACKNOWLEDGMENT}

The research described in this paper was performed in Russian University of Transport and is supported by the Russian Science Foundation (grant 21-19-00023).

\section{References}

[1] H. Xia, N. Zhang, and W. Guo, "Dynamic Interaction of Train-Bridge Systems in High-Speed Railways. Theory and Applications." Beijing: Beijing Jiaotong University Press and Springer-Verlag GmbH, 2018.

[2] W. Zhai, Z. Han, Z. Chen, L. Ling and S. Zhu, "Traintrack-bridge dynamic interaction: a state-of-the-art review," Vehicle Syst. D. pp. 1-44, 2019, DOI: 10.1080/00423114.2019.1605085. 
[3] Y. Yang, J. Yau and Y. Wu, "Vehicle-bridge interaction dynamics: with applications to high-speed railways." Singapore: World Scientific Publishing Co. Pte. Ltd., 2004.

[4] Sufen Li, "Short-Term Load Forecasting of Power System Based on Improved BP Neural Network", Int. J. of Circuits, Systems and Signal Processing, vol.14, pp.840846,2020

[5] Xinhua Duan, "Application of Deep Learning in Power Load Analysis", Int. J. of Circuits, Systems and Signal Processing, vol.14, pp.726-735, 2020

[6] Wei Huang, "Power system Frequency Prediction after Disturbance Based on Deep Learning", Int. J. of Circuits, Systems and Signal Processing, vol.14, pp.716-725, 2020

[7] Xiaoyong Zhu and Hua Zhang, "A Lean Green Implementation Evaluation Method Based on Fuzzy Analytic Net Process and Fuzzy Complex Proportional Assessment", Int. J. of Circuits, Systems and Signal Processing, vol.14, pp.646-655, 2020

[8] N. Samarinas, C. Tzimopoulos and C. Evangelides, "Fuzzy Numerical Solution to Horizontal Infiltration", Int. J. of Circuits, Systems and Signal Processing, vol.12, pp.325-332, 2018

[9] Ning Li, Wenrui Hao, Jiangming Kan, "Landing position prediction model for hydraulic monitors based on the genetic BP neural network", Int. J. of Circuits, Systems and Signal Processing, vol.12, pp.240-245, 2018

[10] Tingting Ye, "Research on the Risk Crisis Prediction of Enterprise Finance by Genetic Algorithm", Int. J. of Circuits, Systems and Signal Processing, vol.12, pp.319324, 2018

[11] Guobing Fan, Chen Guo and Sha Fu, "Numerical optimization solution of system of non-linear equations based on interval algorithm", Int. J. of Circuits, Systems and Signal Processing, vol.12, pp.298-304, 2018

[12] Vladimir Poliakov, "The artificial intelligence and optimization of multibody dynamical system with predicted dynamic behavior", Proceedings of the 24th International Conference on Circuits, Systems, Communications and Computers, Chania, Crete Island, Greece, July 19-22, 2020

[13] J. McCarthy. "Programs with common sense". 1959. Available: http://www-formal.stanford.edu/jmc/

[14] J. McCarthy. "What is artificial intelligence?". 2007. Available: http://www-formal.stanford.edu/jmc/

[15] Luo, X., Qin, Y., (2018), Hybrid Machining: Theory, Methods, and Case Studies. Academic Press, London, UK.

[16] Sarkar, K., Ganguli, R. and Elishakoff, I., 2016. Closedform solutions for non-uniform axially loaded Rayleigh cantilever beams. Struct. Eng. Mech, 60(3), pp.455-470.

[17] Kundu, B. and Ganguli, R., 2020. Closed-form solutions of non-uniform axially loaded beams using Lie symmetry analysis. Acta Mechanica, 231(11), pp.4421-4444.
[18] V. Poliakov, "Optimization facilities for bridges and track on high speed railways." Ingeneria Ferroviaria, Transp. Sc. and Econ. J, Bologna, vol. 3, pp.191-205, March 2018.

[19] V. Poliakov. "Interaction optimization in multibody dynamic system," Int. J. of Theor. and App. Mech., vol. 2, pp. 43-51, 2017

[20] E. Haug, and J. Arora, "Applied Optimal Design. Mechanical and Structural Systems." New York: John Wiley and sons, 1979.

[21] E. Haug, K. Choi, V. Komkov, "Design sencityvity Analisys of Structural Systems." New York: Academic Press, Inc., 1986.

[22] R. Ghiamat, M. Madhkhan and T. Bakhshpoori, "Optimal operators of genetic algorithm in optimizing segmental precast concrete bridges superstructure," Int. J. Optim. Civil Eng., vol. 9, pp. 651-670, 2019.

[23] Z. Aydin, Y. Ayvaz, "Optimum topology and shape design of prestressed concrete bridge girders using a genetic algorithm," Struct. Multidisc. Optim., vol. 41, pp. 151-162, 2010.

[24] M. Alqedra, M. Arafa and M. Ismail, "Optimum Cost of Prestressed and Reinforced Concrete Beams using Genetic Algorithms", J. of Artificial Intelligence, vol. 4, pp. 76-88, 2011.

[25] Maurice Clerc. Standard Particle Swarm Optimisation. 2012. Available: https://hal.archives-ouvertes.fr/hal$\underline{00764996}$

[26] EN 1990 (2002) (English): Eurocode - Basis of structural design. - Brussels: European committee for standardization, 2004

[27] V. Poliakov and V. Saurin, "The stationary optimal control in combined discrete and continuous system," 15th International Conference on Stability and Oscillations of Nonlinear Control Systems (Pyatnitskiy's Conference), 2020, doi: 10.1109/STAB49150.2020.9140605.

[28] V. Poliakov, Zhang Nan, V. Saurin and Dang Ngok Thanh. Running Safety of a High-Speed Train within a Bridge Zone, International Journal of Structural Stability and Dynamics, Vol.20(11) (13 pages), 2020, doi: $10.1142 / \mathrm{S} 0219455420501163$

\section{Creative Commons Attribution License 4.0 (Attribution 4.0 International, CC BY 4.0)}

This article is published under the terms of the Creative Commons Attribution License 4.0 https://creativecommons.org/licenses/by/4.0/deed.en_US 